\title{
Trade-Offs between Growth Rate, Sporulation and Pathogenicity in Verticillium dahliae
}

\author{
Ahmed A. ElSharawy ${ }^{1,2}$, Xiaoping $\mathrm{Hu}^{1} \&$ Jiarong Yang ${ }^{1}$ \\ ${ }^{1}$ State Key Laboratory of Crop Stress Biology for Arid Areas, College of Plant Protection, Northwest A\&F \\ University, Yangling, China \\ ${ }^{2}$ Department of Plant Production, Faculty of Environmental Agricultural Sciences, Suez Canal University, Egypt \\ Correspondence: Jiarong Yang, State Key Laboratory of Crop Stress Biology for Arid Areas, College of Plant \\ Protection, Northwest A\&F University, Yangling 712100, China. Tel: 86-133-5920-1326. E-mail: \\ yjrong_soilborne@hotmail.com; yjrong@nwsuaf.edu.cn
}

Received: April 1, 2015 Accepted: May 5, 2015 Online Published: June 15, 2015

doi:10.5539/jas.v7n7p35 URL: http://dx.doi.org/10.5539/jas.v7n7p35

\begin{abstract}
Verticillium dahliae is a soil-borne phytopathogenic fungus that causes vascular wilt diseases in cotton (Gossypium hirsutum L.). We have illustrated the correlations between growth rate, sporulation and pathogenicity in $V$. dahliae mutants. A total of $196 \mathrm{~V}$. dahliae mutants generated by Agrobacterium tumefaciens-mediated transformation, was used throughout this study. Compared with a control strain, the mutants could be significantly grouped into three types by differentiation with to that of control strain. The results indicated that linear regression analysis of the relationship was performed and the gradient and intercept were shown for growth rate and disease index. The correlation coefficient was observed if the infection is high. When their correlation of the growth rate is high, the growth rate and sporulation quantity of mutants had significantly a power correlation. This study, which may play an important role in the population structure of the pathogen, highlights fundamental biological differences between the mutants involving different infection strategies.
\end{abstract}

Keywords: Verticillium dahlia, growth rate, sporulation, pathogenicity

\section{Introduction}

Verticillium dahliae Kleb is a soilborne fungal pathogen that belongs to Ascomycetes and causes vascular wilt in a broad range of dicotyledonous host plants (Pegg \& Brady, 2002; Shuai et al., 2014; Xiao \& Subbarao, 1998). A list of infected hosts by the pathogen is continually expanding as disease outbreaks on new hosts which are identified (Bishop \& Cooper, 1983; Garas, Wilhem, \& Sagen, 1986; Wilhelm, 1955). Serious outbreaks due to $V$. dahliae can cause severe yield losses in crops such as cotton, cauliflower and sunflower (Bhat, Smith, Koike, Wu, \& Subbarao, 2003; Fradin et al., 2011; Gao et al., 2013). Once the plant is diagnosed with Verticillium wilt since then there is no fungicide that can cure it (Fradin \& Thomma, 2006). Three vegetative phases make up $V$. dahliae's life cycle, i.e., dormant, parasitic and saprophytic and no sexual life cycle is known so far (Klimes \& Dobinson, 2006). The broad host range and long-term survival of microsclerotia in soil make this pathogen difficult to manage in agricultural systems. Moreover, $V$. dahliae is known to disperse by the movement of soil, irrigation water and with seed and vegetative propagation (Koike, Subbarao, Davis, Gordon, \& Hubbard, 1994). Some clonal lineages have been dispersed across broad geographic distances (Conn, Tenuta, \& Lazarovits, 2005) by agriculture. Physical and chemical factors have pronounced effective control on this disease that depend on the understanding of the complex factors governing the epidemiological process of this pathogen such as growth rate and sporulation (on artificial media which are important biological characteristics), pathogenicity and their interactions. Till today, all these aspects are still under study and the pathogen population is not well characterized and little is known about its prevalence in Verticillium dahliae. Morphological characteristics of fungal pathogens are studied at both asexual and sexual stages for the identification of these pathogens. In the case of some fungal pathogens, specific conditions are provided for sporulation (production of spores/conidia) and the morphological criteria are determined for appropriate placement in different species, genera, family etc. (Debode et al., 2007; Fradin et al., 2009). In the present study, we are comparing the growth rate, sporulation and pathogenicity of $V$. dahliae and its mutants. Further, we also measure these mutants in vitro and examine them for possible correlations. 


\section{Materials and Methods}

\subsection{Fungal Isolates and Culture Conditions}

A total of 196 mutants were generated from virulent defoliating Verticillium dahliae strains in cotton (Figure 1) by Agrobacterium tumefaciens-mediated transformation method described by Maruthachalam et al. (2008) and that was followed with some modifications. These mutants were inoculated onto PDA plates and incubated for 7-10 days at $25{ }^{\circ} \mathrm{C}$ in the dark until colonies developed. Single-spore isolates of $V$. dahliae were obtained, identified as previously described (Goud, Termorshuizen, \& Gams, 2003; Hawksworth \& Talboys, 1970) and maintained on PDA medium in tubes at $10{ }^{\circ} \mathrm{C}$ for further use. Then these mutants were confirmed by PCR analysis with $h p h$-specific primers (data not shown). The isolates were stored in microconidial suspension in 25 $\%$ glycerol at $-80{ }^{\circ} \mathrm{C}$ till they were used.

\subsection{Growth Rate and Sporulation}

To measure the growth rate and sporulation in vitro, we were selected the Czapek Dox and PDA as basic medium (Zhang et al., 2012). The $\mathrm{pH}$ of the test media were maintained at 5.5 and which were optimal for the growth and sporulation in majority of fungi. Wild type and its mutants were inoculated and grown on the PDA plates in the dark at $25 \pm 1^{\circ} \mathrm{C}$ and the growth rates were subsequently calculated based on the colony size. The difference in colony diameter of two adjacent days was determined to represent the colony growth rate. Disks with $6 \mathrm{~mm}$ diameter were taken from the edge of colonies and placed onto the center of PDA plates and each mutant was analyzed three times with three replicate. All cultures were incubated at $25 \pm 1{ }^{\circ} \mathrm{C}$ for $5-7$ days in the dark. Each value indicates the mean and standard error.

The spore production quantification of the wild type and mutants were assessed by placing drops of a spore suspension through two ways: Firstly, by using the Petri-dish with a sharp spatula and washing several times with a total volume of $100 \mathrm{~mL}$ of sterilized water. The suspension was filtered through a plastic sieve to separate the spores from the mycelium. Secondly, by using the conidial suspension through adding $200 \mu 1$ of the conidial suspension of $1 \times 10^{6}$ conidia/ml to $50 \mathrm{ml}$ of medium and incubating the cultures at $25^{\circ} \mathrm{C}$, by vigorous shaking $150 \mathrm{rpm}$ for 7 days. To quantify the final conidia concentration of spore, a hemocytometer under a microscope was used. Also here each value indicates the mean and standard error. The linear growth and number of spores produced by each replicate was recorded after 7 days of incubation.

\subsection{Pathogenicity Test}

Cotton plants (cv. Ji Mian No. 11) were used in the pathogenicity test of wild type and its mutants. A root-dip method was employed for plant inoculation (Gao et al., 2010). Cotton seeds were sterilized on their surface with $70 \%$ ethanol for 3-5 min, then with $2 \%$ sodium hypochlorite solution $(\mathrm{v} / \mathrm{v})$ for $15 \mathrm{~min}$, and then washed three times under sterile water and dried between filter paper before sowing. Seedlings were grown in pots containing disinfested soil and compost mixture $(1: 1, \mathrm{v} / \mathrm{v})$ under glass house conditions till the two true-leaf development stages. The roots of three weeks age plants were trimmed just before inoculation (Joaquim \& Rowe, 1991). Three pots were used for each isolate and every pot contained 5 plants (a total of 15 plants per isolate). Inoculums were harvested from 1-week old liquid cultures on PDB (Potato Dextrose Broth), filtrated using four layers of gauze, and washed with sterilized water twice.

The pathogenicity tests were performed by dipping the plant roots into a conidial suspension $1 \times 10^{6}$ spores $/ \mathrm{ml}$ which was determined by a hemacytometer under a microscope (Olympus BX51, Japan). Pots were fertilized with the recommended dose of organic and inorganic fertilizers and watered regularly. Fifteen un-inoculated seedlings were dipped in sterile distilled water as control strain. Disease symptoms appeared after 7 days, disease severity was observed and recorded as described by Hu et al. (2013). The experiment was repeated once. 

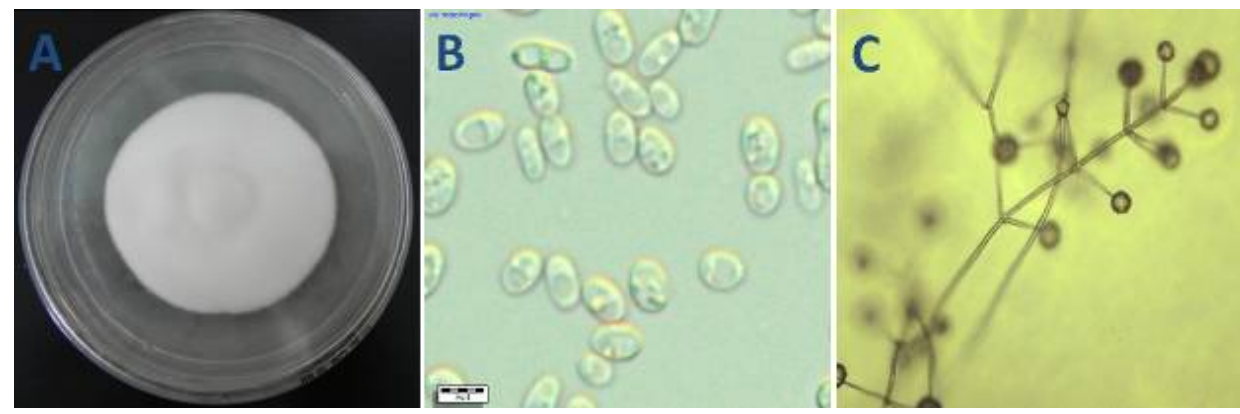

Figure 1. (A) front view of colony morphology on PDA for Wild type; (B) a single spore produced by wild type;

(C) branched conidiophores, which form whorls capped with flask-shaped and pointed phialides carrying terminal

\subsection{Data Analysis}

All experiments were conducted in three replications and repeated 3 times, and the SPSS software was used to analyze the data (Standard Version 16.0, SPSS Inc., Shanghai, China). All of the values are expressed as the mean \pm the standard error (SE). The significance of differences was determined by one-way analysis of variance (ANOVA) with Student-Newman-Keuls (S-N-K) and the differences with $P$ values of $<0.05$ were considered statistically significant.

\section{Results}

\subsection{Growth Rate}

The growth rate of colonies was compared on PDA medium and recorded the highest mycelia diameters after 7 days.In present study the results were subjected to analyze of variance tests at $P<0.05$ to compare the growth rate between the wild type and its mutant. We observed that they were significantly divided into three groups. Based on their colony growth rate, $35.2 \%$ of mutants was significantly reduced the growth rate whereas, about Also $34.7 \%$ of mutants was increased their growth rate. No significant difference was found among $30.1 \%$ because they had similar growth with the wild type (Figure 2A).

\subsection{Sporulation Quantity}

In this trail an initial spore concentration of $1 \times 10^{6}$ conidia $\mathrm{ml}^{-1}$ was cultured on the PDA medium. Figure 2B showed that, the sporulation quantities of wild strain and its mutants were determined. The sporulation rate was significantly influenced compared with the wild type. The sporulation quantity of $42.9 \%$ mutants was much higher than that the wild type, whereas the $30.1 \%$ of mutants was recorded lower than that of the wild type. And $27 \%$ had similar sporulation quantity to that of wild type strain.

\section{- Higher}

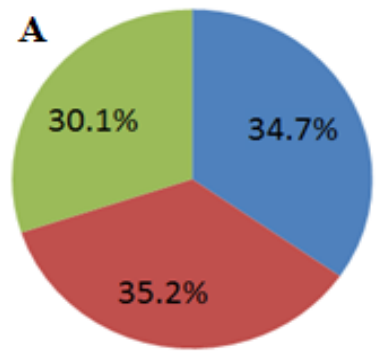

Lower

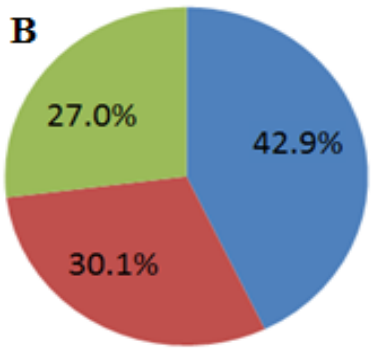

Similar

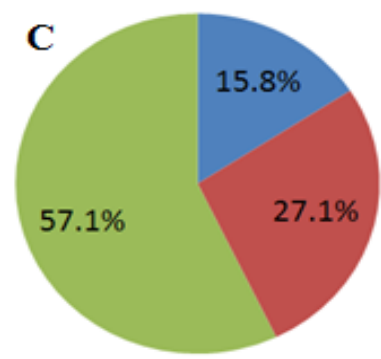

Figure 2. Comparison between the wild type strain as a control and its mutants, the mutants could be significantly grouped into three types by differentiation with to that of control strain. (A) Growth rate. (B) Sporulation quantity.

(C) Pathogenicity test 


\subsection{Pathogenicity Test}

Pathogenicity test was performed using the conidia from wild-type strain with cotton cultivar Ji Mian NO11. Infections were performed independently for a period of up to 6wpi to examine the pathogenicity of different genotypes. Symptoms could be observed obviously, including leaf chlorosis and stunting in plants that infected with the wild type and its mutants at $21 \mathrm{dpi}$. Based on the disease severity, the 196 mutants were significantly divided into three groups which were compared with the wild type. We found out that $15.8 \%$ and $27.1 \%$ of mutants were higher and lower than that the wild type in pathogenicity respectively. Meanwhile $57.1 \%$ of mutants was similar to that of the wild type in pathogenicity (Figure 2C). Table 1 showed that, Comparison between wild type and 25 mutants randomly selected from our experiment results which were measured that the growth rate and sporulation in vitro and the pathogenicity in vivo.

\subsection{The Correlations between Growth Rate, Sporulation and Pathogenicity}

In regression analysis, A highly significant correlations were found between the growth rate on the fresh PDA medium of each mutant and the disease index recorded at $21 \mathrm{dpi}\left(\mathrm{R}^{2}=0.9129\right)$, the gradient was 15.54 , and the intercept was 48.114. They all were particularly useful for obtaining a linear relationship and explaining a reasonably high percentage of the variation in colony size indicating that the percentage of the variation in disease symptoms. Based on $\left(\mathrm{R}^{2}=0.8936\right)$, the greatest amount of the sporulation quantity and percentage of the variation in colony size had a significantly power correlations with 2.8532 intercept power and with the gradient of 0.012 . Our comparison clearly indicates that the growth rate is the key factor for pathogenicity, especially when sporulation is lower than that of control strain (Figure 3).

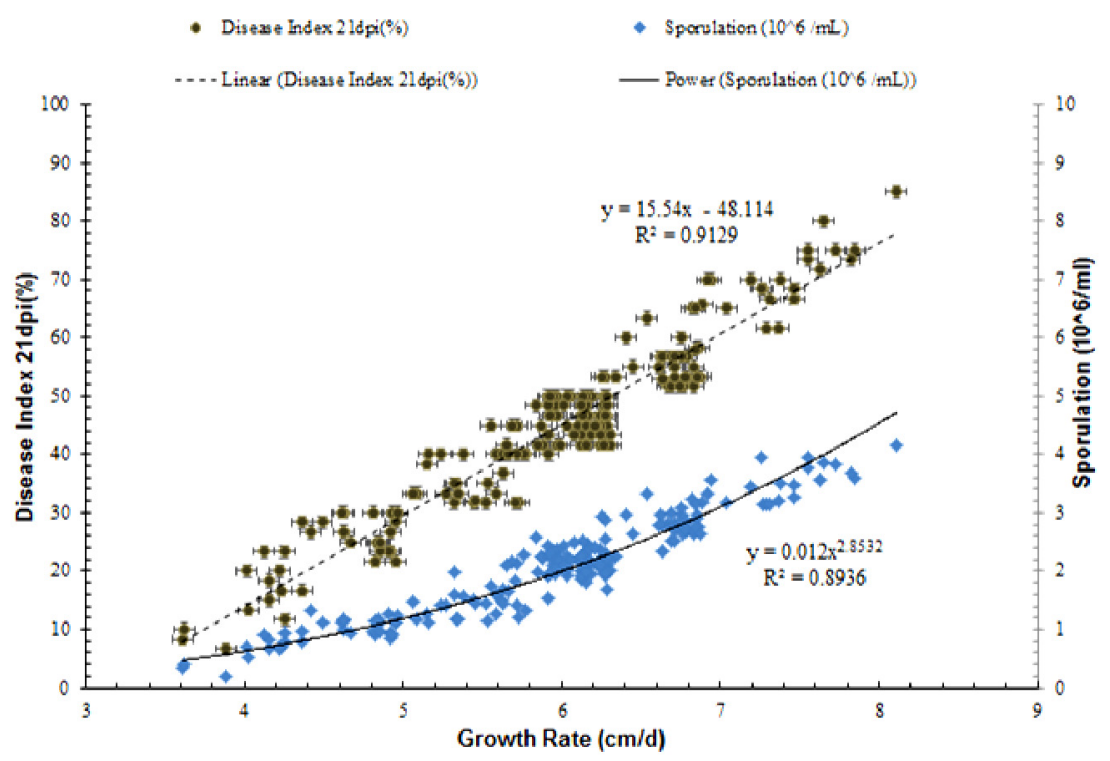

Figure 3. The correlations between growth rate, sporulation and pathogenicity

\section{Discussion}

Verticillium wilt is typically soil-borne disease. The speed of mycelia growth, the number of spore-bearing and disease index had effects on each other. In our experiment the trade-offs between growth rate, sporulation and pathogenicity in vitro/in vivo experiments on $V$. dahliae wild type and 196 mutants have been carried out. The study of the growth rate and sporulation in vitro are markedly influenced by comparing with the wild type.

Screening of 196 mutants was distinct in providing virtually complete coverage of the value of the molecular mechanisms that involved of $V$. dahliae indirectly by correlations between biological properties. Overall between-the wild type and its mutant trade-offs in performance of multiple-host pathogens are expected to increase under stabilizing selection as the number of hosts and their phylogenetic distance increase in a plant community (Maruthachalam et al., 2008b).

The lifestyle of $V$. dahliae in its center of origin might be shaped by the constraint of short-distance spore dispersal in a high diversity species forest and the fitness costs caused by the need for a multiple-host strategy associated with reduced sporulation. 
Such reduced sporulation, however, could be enough for the effective spread of $V$. dahliae in areas where environmental conditions are similar to those expected in its native habitat.

During the study the behavior for $V$. dahliae in terms of growth rate, sporulation and pathogenicity were revealed. In addition, this contrasting behavior for $V$. dahliae underline the presence of probably two infection strategies; the first is based on sporulation for better dispersion of the pathogen and the second is based on growth rate for rapid tissues and vessel colonization. In fact, aggressiveness in vivo and in vitro tests revealed the best growth rate and the highest disease index. These differences can be explained by differential mutant sensitivity to mycostasis. This has been investigated as a mechanism by which propagules are protected from spontaneous germination in the absence of potentially colonisable substrata (Liu et al., 2013).

In the determination of virulence and sporulation, the sporulation was reduced; however, it could be effective to spread the $V$. dahliae and will not have any difference in its virulence. Plants were subsequently grown under greenhouse conditions for up 16 weeks (isolate comparison study) or 3 to 4 weeks (rapidly study). This assay is preferred because it is rapidly compared with the conventional greenhouse assay in the soil medium (Klosterman \& Hayes, 2009). Conventional methods for pathogenicity are laborious and time consuming, and require 3 to 4 weeks for final results.

Our interesting study is for correlating between growth rate, sporulation and pathogenicity in $V$. dahliae mutants. According to our results, 'growth rate' and 'sporulation' strategies are more prevalent in $V$. dahliae in cotton, respectively. Evolutions in the pathogen population structure at one hand and distribution of the disease in the field in time and space on the other hand are required to be kept under investigation for a better disease management.

Table 1. Comparison between the growth rate, sporulation and pathogenicity of wild type and some mutants

\begin{tabular}{llll}
\hline Mutant & Disease Index 21 dpi & Growth Rate $(\mathrm{cm} / \mathrm{d})$ & Sporulation $\left(10^{6} / \mathrm{ml}^{-1}\right)$ \\
\hline Wild type & 56.67 & $6.96 \pm 0.12$ & $2.10 \pm 0.10$ \\
M10 & 20.00 & $7.22 \pm 0.05$ & $2.21 \pm 0.08$ \\
M17 & 26.67 & $6.63 \pm 0.25$ & $1.80 \pm 0.02$ \\
M65 & 25.00 & $6.67 \pm 0.09$ & $2.20 \pm 0.04$ \\
M49 & 15.00 & $5.76 \pm 0.16$ & $1.57 \pm 0.06$ \\
M73 & 31.67 & $6.12 \pm 0.12$ & $1.15 \pm 0.05$ \\
M90 & 46.67 & $6.45 \pm 0.35$ & $1.93 \pm 0.04$ \\
M94 & 8.33 & $7.61 \pm 0.63$ & $0.85 \pm 0.10$ \\
M99 & 70.00 & $6.78 \pm 0.15$ & $2.20 \pm 0.11$ \\
M89 & 41.67 & $6.88 \pm 0.19$ & $1.90 \pm 0.08$ \\
M128 & 55.00 & $7.11 \pm 0.19$ & $2.01 \pm 0.04$ \\
M185 & $8.03 \pm 0.23$ & $1.63 \pm 0.08$ \\
M129 & 13.33 & $5.46 \pm 0.09$ & $1.68 \pm 0.04$ \\
M77 & 16.67 & $7.83 \pm 0.12$ & $2.00 \pm 0.07$ \\
M109 & 31.67 & $6.95 \pm 0.23$ & $2.08 \pm 0.10$ \\
M155 & 60.00 & $5.66 \pm 0.16$ & $1.48 \pm 0.08$ \\
M147 & 11.67 & $7.11 \pm 0.18$ & $1.76 \pm 0.09$ \\
M139 & 85.00 & $5.63 \pm 0.10$ & $2.05 \pm 0.07$ \\
M25 & 71.67 & $6.56 \pm 0.17$ & $1.66 \pm 0.10$ \\
M67 & 48.33 & $6.62 \pm 0.05$ & $2.35 \pm 0.07$ \\
M75 & 40.00 & $4.88 \pm 0.12$ & $0.90 \pm 0.07$ \\
M140 & 6.67 & $7.82 \pm 0.38$ & $2.13 \pm 0.06$ \\
M190 & 21.67 & & $1.90 \pm 0.20$ \\
\hline
\end{tabular}




\begin{tabular}{llll}
\hline M55 & 66.67 & $6.31 \pm 0.08$ & $2.10 \pm 0.10$ \\
M138 & 45.00 & $7.60 \pm 0.10$ & $2.16 \pm 0.10$ \\
M47 & 53.33 & $6.61 \pm 0.06$ & $2.21 \pm 0.12$
\end{tabular}

Note. M: Mutant, dpi: days post inoculation. The values in the table above were the average of three replicates for wild-type and its mutants, respectively, and the value following by " \pm " was standard error. Values were significantly different $(P<0.05)$ in S.N.K test of one-way ANOVA.

\section{Acknowledgements}

This research was supported by NSFC (31371888) and by 111 Project from Education Ministry of China (B07049).

\section{References}

Bhat, R., Smith, R., Koike, S., Wu, B., \& Subbarao, K. (2003). Characterization of Verticillium dahliae isolates and wilt epidemics of pepper. Plant disease, 87(7), 789-797. http://dx.doi.org/10.1094/pdis.2003. 87.7.789

Bishop, C., \& Cooper, R. M. (1983). An ultrastructural study of root invasion in three vascular wilt diseases. Physiological Plant Pathology, 22(1), 15-27. http://dx.doi.org/10.1016/s0048-4059(83)81034-0

Conn, K. L., Tenuta, M., \& Lazarovits, G. (2005). Liquid swine manure can kill Verticillium dahliae microsclerotia in soil by volatile fatty acid, nitrous acid, and ammonia toxicity. Phytopathology, 95(1), 28-35. http://dx.doi.org/10.1094/phyto-95-0028

Debode, J., Maeyer, K. D., Perneel, M., Pannecoucque, J., Backer, G. D., \& Höfte, M. (2007). Biosurfactants are involved in the biological control of Verticillium microsclerotia by Pseudomonas spp. Journal of applied microbiology, 103(4), 1184-1196. http://dx.doi.org/10.1111/j.1365-2672.2007.03348

Fradin, E. F., \& Thomma, B. P. (2006). Physiology and molecular aspects of Verticillium wilt diseases caused by $V$. dahliae and $V$. albo-atrum. Molecular Plant Pathology, 7(2), 71-86. http://dx.doi.org/10.1111/j.1364-3703.2006.00323

Fradin, E. F., Abd-El-Haliem, A., Masini, L., van den Berg, G. C., Joosten, M. H., \& Thomma, B. P. (2011). Interfamily transfer of tomato $\mathrm{Ve} 1$ mediates Verticillium resistance in Arabidopsis. Plant physiology, 156(4), 2255-2265. http://dx.doi.org/10.1104/pp.111.180067

Fradin, E. F., Zhang, Z., Ayala, J. C. J., Castroverde, C. D., Nazar, R. N., Robb, J., \& Thomma, B. P. (2009). Genetic dissection of Verticillium wilt resistance mediated by tomato Ve1. Plant physiology, 150(1), 320-332. http://dx.doi.org/10.1104/pp.109.136762

Gao, F., Zhou, B.-J., Li, G.-Y., Jia, P.-S., Li, H., Zhao, Y.-L., \& Guo, H.-S. (2010). A glutamic acid-rich protein identified in Verticillium dahliae from an insertional mutagenesis affects microsclerotial formation and pathogenicity. PLoS ONE, 5(12), e15319. http://dx.doi.org/10.1371/journal.pone.0015319

Gao, W., Long, L., Zhu, L.-F., Xu, L., Gao, W.-H., Sun, L.-Q., \& Zhang, X.-L. (2013). Proteomic and virus-induced gene silencing (VIGS) analyses reveal that gossypol, brassinosteroids, and jasmonic acid contribute to the resistance of cotton to Verticillium dahliae. Molecular \& Cellular Proteomics, 12(12), 3690-3703. http://dx.doi.org/10.1074/mcp.m113.031013

Garas, N., Wilhem, S., \& Sagen, J. (1986). Relationship of cultivar resistance to distribution of Verticillium dahliae in inoculated cotton plants and to growth of single conidia on excised stem segments. Phytopathology, 76(10), 1005-1010. http://dx.doi.org/10.1094/phyto-76-1005

Goud, J.-K. C., Termorshuizen, A. J., \& Gams, W. (2003). Morphology of Verticillium dahliae and V. tricorpus on semi-selective media used for the detection of $V$. dahliae in soil. Mycological Research, 107(07), 822-830. http://dx.doi.org/10.1017/s0953756203008050

Hawksworth, D., \& Talboys, P. (1970). Verticillium dahliae CMI descriptions of pathogenic fungi and bacteria No. 256. Wallingford, UK: CAB International.

Hu, X., Bai, Y., Chen, T., Hu, D., Yang, J., \& Xu, X. (2013). An optimized method for in vitro production of Verticillium dahliae microsclerotia. European Journal of Plant Pathology, 136(2), 225-229. http://dx.doi.org/10.1007/s10658-013-0170-2 
Joaquim, T. R., \& Rowe, R. C. (1991). Vegetative compatibility and virulence of strains of Verticillium dahliae from soil and potato plants. Phytopathology, 81(5), 552-558. http://dx.doi.org/10.1094/phyto-81-552

Klimes, A., \& Dobinson, K. F. (2006). A hydrophobin gene is involved in microsclerotial development and spore viability in the plant pathogen Verticillium dahliae. Fungal Genetics and Biology, 43(4), 283-294. http://dx.doi.org/10.1016/j.fgb.2005.12.006

Klosterman, S. J., \& Hayes, R. J. (2009). A soilless Verticillium wilt assay using an early flowering lettuce line. Plant disease, 93(7), 691-698. http://dx.doi.org/10.1094/PDIS-93-7-0691

Koike, S. T., Subbarao, K. V., Davis, R. M., Gordon, T. R., \& Hubbard, J. C. (1994). Verticillium wilt of cauliflower in California. Plant disease, 78(11), 1116-1121. http://dx.doi.org/10.1094/pd-78-1116

Liu, L., Zhao, D., Zheng, L., Hsiang, T., Wei, Y., Fu, Y., \& Huang, J. (2013). Identification of virulence genes in the crucifer anthracnose fungus Colletotrichum higginsianum by insertional mutagenesis. Microbial Pathogenesis, (64), 6-17. http://dx.doi.org/10.1016/j.micpath.2013.06.001

Maruthachalam, K., Nair, V., Rho, H.-S., Choi, J., Kim, S., \& Lee, Y. H. (2008). Agrobacterium tumefaciens-mediated transformation in Colletotrichum falcatum and C. acutatum. Journal of Microbiology and Biotechnology, 18(2), 234-241.

Pegg, G. F., \& Brady, B. L. (2002). Verticillium wilts. Wallingford, UK: CABI Publishing. http://dx.doi.org/10.1079/9780851995298.0000

Shuai, H., Dongfang, H., Ahmed, A. E., Chunsheng, W., Wenjing, S., Jiarong, Y., \& Xiaoping, H. (2014). Generation of T-DNA Insertional Mutant Library for Defoliating Verticillium dahliae. Acta Agriculturae Boreali-Occidentalis Sinica, 9, 128-134.

Wilhelm, S. (1955). Longevity of the Verticillium wilt fungus in the laboratory and field. Phytopathology, 45(5), 180-181.

Xiao, C., \& Subbarao, K. (1998). Relationships between Verticillium dahliae inoculum density and wilt incidence, severity, and growth of cauliflower. Phytopathology, 88(10), 1108-1115. http://dx.doi.org/10.1094/phyto.1998.88.10.1108

Zhang, B., Yang, Y., Chen, T., Yu, W., Liu, T., Li, H., \& Liu, L. (2012). Island cotton Gbvel gene encoding a receptor-like protein confers resistance to both defoliating and non-defoliating isolates of Verticillium dahliae. PLOS ONE, 7(12), e51091. http://dx.doi.org/10.1371/journal.pone.0051091

\section{Copyrights}

Copyright for this article is retained by the author(s), with first publication rights granted to the journal.

This is an open-access article distributed under the terms and conditions of the Creative Commons Attribution license (http://creativecommons.org/licenses/by/3.0/). 